\title{
Kaposi sarcoma appearing 20 year post renal transplant
}

\section{Albitar Yassir and Amir E. Ali}

Abstract: A kidney- transplanted Saudi patient presented with a skin rash for which he was treated as fungal infection. The patient developed these lesions 20 years after transplantation. The clinical picture was that of Kaposi's sarcoma which was confirmed by histopathology. The patient developed these lesions after he was shifted to prednisolone and mycophenolate mofetil after four years of prednisolone, azathioprine and cyclosporine immunosuppressive therapy.

Key words: Kaposi's sarcoma, mycophenolate mofetil, cyclosporine.

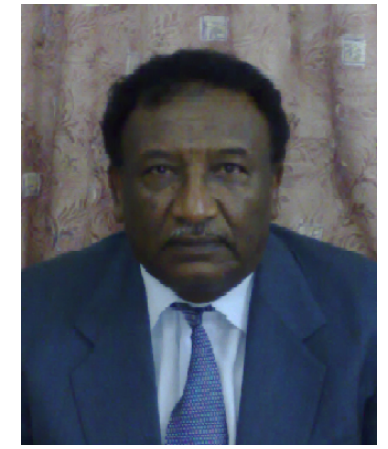

$\mathrm{S}$ arcoma is a cancer that develops in connective tissues such as cartilage, bone, muscle, blood vessel or fibrous tissue. In 1872 Moritz Kaposi first described "Idiopathisches multiple pigmentsarkom der Haut" which has become known as Kaposi sarcoma $(\mathrm{KS})^{1}$. It was postulated that Kaposi sarcoma, especially in patients with advanced skin tumors are reactive proliferations rather than true malignancies with metastatic dissemination ${ }^{2}$.

KS has several types: Classic KS which was considered a rare disease that mostly affects elderly men of Mediterranean or Jewish heritage, African KS (Endemic) including the fatal infantile $\mathrm{KS}$, Transplant-Related $\mathrm{KS}$ (Acquired) and AIDS-Related (Epidemic) Kaposi sarcoma ${ }^{3}$.

In the last 20 years most of $\mathrm{KS}$ cases were associated with HIV infection

It was found that KS risk is high in immunosuppressed transplant recipients, and most cases occurred in the first two years after transplantation. The risk increases steadily with recipient age, and in males ${ }^{4}$.

KS is $150-200$ times more likely to develop in transplanted patients than in general population. There is strong evidence that KS develops in recipients with pre-existing infection with human herpes virus $8(\mathrm{HHV}-8)^{3,4}$. According to Tessari $\mathrm{etal}^{5}$ only combined immunosuppressive therapy with mycophenolate mofetil + cyclosporine + prednisolone were independently associated with KS.

\footnotetext{
Department of Dermatology, King Abdul Aziz Hospital, Holly Makkah, Saudi Arabia.Correspondance to : Prof. Amir E Ali P O Box 7607 , Faculty of medicine ,Umm Alqurra University, Holly Makkah. Saudi Arabia amirelmubarek@excite.com
}

\section{Case report:}

A 45 -years- old man was seen in post renal transplant clinic with multiple skin lesions. The lesion were diagnosed initially and treated as fungal infection (Tinea corporis). The patient had renal transplant 20 years prior to this presentation. $\mathrm{He}$ was on immunosuppressive therapy. Firstly, he was on prednisolone, azathioprine and cyclosporin for four years, and then shifted to prednisolone plus mycophenolate mofetil only. He was on regular follow up by the renal unit.

Clinical evaluation of the patient revealed a large violet patch $(15 \times 10 \mathrm{~cm})$ over the surface of the right lower half of the abdomen (see figure 1)

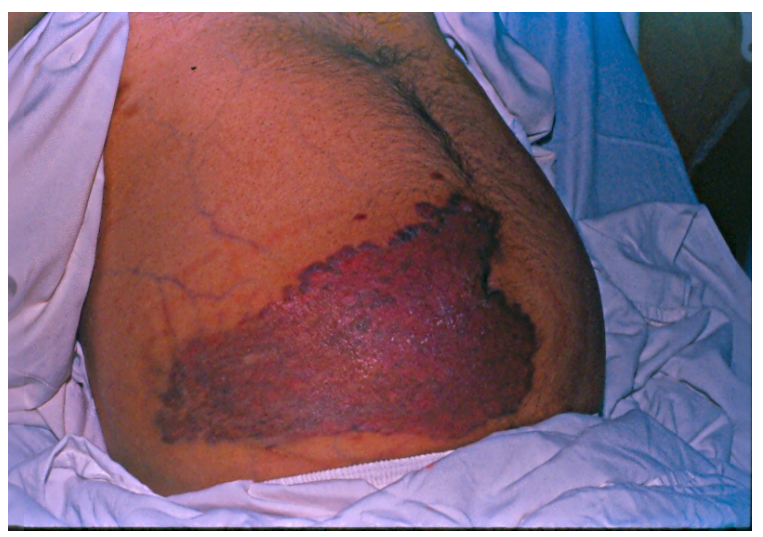

Figure(1) shows a large $(15 \mathrm{x} ! 0 \mathrm{xcm})$ violet patch on the right lower half of the abdomen.

There were also violet multiple nodules seen over the upper part left chest over the left breast (figure 2) and over the surgical incision. 
Figure(2) shows multiple nodules over the left breast.

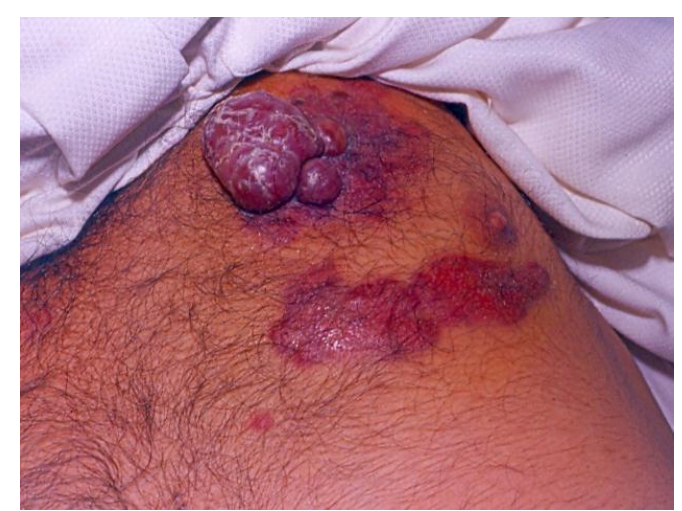

In addition, there are two violet patches $(2 \times 3 \mathrm{~cm}$ each) seen on the lateral side of the right upper arm and another patch on the right thigh.

Blood tests revealed normal white blood cells count (11000), platelet count (157) but a high ESR $(45 \mathrm{~mm} / \mathrm{hr})$, a low haemoglobin (7.2 $\mathrm{gm} / \mathrm{dl})$, a high blood urea $(71 \mathrm{mg} / \mathrm{dl})$ and a high creatinine $(2.0 \mathrm{mg} / \mathrm{dl})$ otherwise normal chemistry.

Sputum was negative for AFB

Serological tests for both HIV and VDRL were negative.

Chest X-ray showed multiple infiltrations with patchy opacities bilaterally in the lower parts of both lungs suggestive of metastasis. (Figure 4)

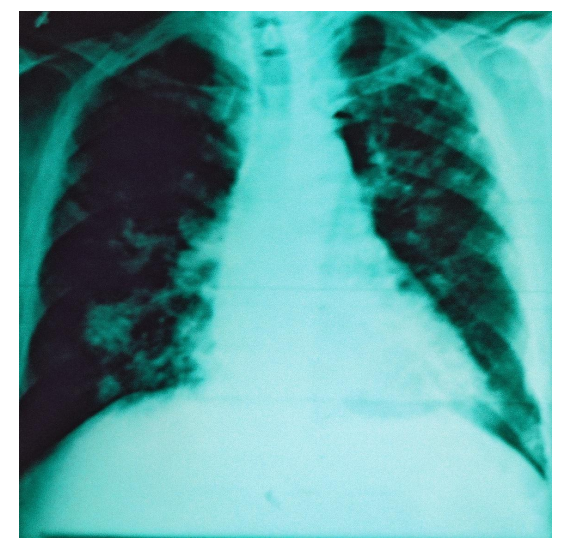

Figure (4) Chest X-ray of the patient showing multiple infiltrations with patchy opacities on the lower parts of lungs.

Ultra sound of the abdomen showed shrunken parental kidney while the transplanted kidney was obscured by gases. Liver was coarse and in-homogenous echo texture with echo-poor area suggestive of (c) Sudan JMS Vol. 3, No. 1, Mar 2008 metastasis while pancreas and spleen were normal and no ascitis was seen. CT scan was recommended but it was not possible to perform before patient's death.

Upper gastrointestinal endoscopy showed ulcerated nodular lesions with bleeding mucosa in the greater curvature and the middle body of the stomach. It was not possible to take a biopsy from these lesions because of the instability of the patient.

The patient was referred to dermatology department where a skin biopsy from the lesions revealed a thinned out epidermis. Dermis and subcutaneous tissue showed spindle shape cells proliferation seen in fascicles with separating slit-like vascular spaced lined by mildly atypical nuclei. There were extravasations of red blood cells and haemosidrin pigmentation. The superficial part showed ill-defined vascular proliferation, newly formed vascular formation and inflammatory infiltrates (Figures5, 6, and 7), these findings were consistent with the diagnosis of Kaposi sarcoma.

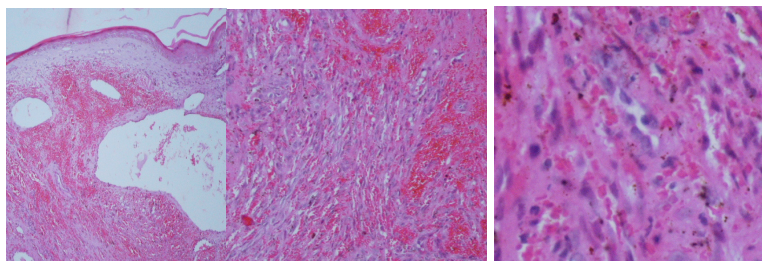

Fig 5,6 and 7 show the histopathogical findings

\section{Discussion:}

$\mathrm{KS}$ is a multifocal vascular tumor that occurs most commonly in patients who have immunosuppression ${ }^{6}$. It is the most common malignancy encountered in renal transplant recipients ${ }^{7}$. It is seen up to 400 times more frequently in transplant patients compared to normal population ${ }^{8}$.

The highest percentage for post renal transplant K.S is reported from Saudi Arabia?. $\mathrm{KS}$ was found to be the most common malignancy encountered in renal transplant recipients in Iraq with a prevalence of $2.9 \%{ }^{7}$. Altaee et al ${ }^{7}$ indicated that the average latency period between transplantation and development of malignancy was 6.5 months for KS. However, Italian organ transplant recipients have an increased risk (about 100 times greater) for Kaposi sarcoma compared 
to general population especially during the first two years after transplantation ${ }^{5}$.

Post renal transplant KS is related more to the immunosuppressive protocol containing cyclosporine compared to azathioprine ${ }^{8}$. It has been suggested that cyclosporine is a risk factor for the development of KS as it has a direct cancer promoting effect by cellautonomous mechanisms ${ }^{10}$.

On the other hand, regression of KS has been reported on replacing cyclosporine by mycophenolate mofetil ${ }^{11}$.

Hussein etal reported disappearance of KS after replacing cyclosporine and azathioprine by mycophenolate mofetil ${ }^{12}$. However, even after the cessation of cyclosporine and azathioprine and replacing them with mycophenolate mofetil in our reported case, KS developed. This might supports earlier reports $^{13}$. It is not very clear whether the introduction of mycophenolate mofetil contributed to the appearance of $\mathrm{KS}$ in our patient or not. Some reports showed a higher $(0.8 \%)$ incidence for developing KS while on mycophenolate mofetil treatment compared with only $0.01 \%$ in patients not on mycophenolate mofetil treatment ${ }^{14}$.

As for the average latency period between transplantation and development of KS, some reports were as low as 6.5 months others gave up to 21 months $^{7,15}$. However it was found that most cases occurred in the first two years after transplantation. Surprisingly our patient had developed KS almost 20 years after the transplantation ${ }^{4}$.

\section{Conclusions:}

It is concluded that renal transplant patients may develop KS even if shifted from cyclosporine to mycophenolate mofetil therapy. Post renal KS can occur up to twenty years after the transplant.

\section{References:}

1. Stănescu L. Foarfă C, Georgescu AC,Georgescu I. Kaposi Sarcoma Associated with AIDS. Rom J Morphol Embryol 2007;48(2):181-7.

2. Duprez R, Lacoste V, Brière $J$ et al. Evidence for multicolonal origin of multicenteric advanced lesions of Kaposi sarcoma. J Nat Cancer Inst 2007;99(14) :1086-94.

3. Fernandes PI, Vazquez TL, Lazaro QM et al Disseminated classic Kaposi's sarcoma. Clin Transl Oncol 2007 Apr;9(4):255-7.

4. Mbulateye SM and Engels EA. Kaposi's sarcoma risk among transplant recipients in the United States (1993-2003). Int J Cancer 2006;. 119(11): 2685-91.

5. Tessari G, Naldi L, Boschiero L et al. Incidence and clinical predictors of Kaposi's sarcoma among 1721 Italian solid organ transplant recipients : a multicenter study. Eur J Dermatol 2006;16(5): 553-7

6. Jessop S. HIV-associated Kaposi's sarcoma .Dermatol Clin 2006;24(4):509-20.

7. Altaee IK, Jaleel NA, Aljubury HM et al. Incidence and types of malignancies in renal transplant recipients in Iraq. Saudi J Kidney Dis Transpl 2006; 17(3): 40814.

8. Frances C. Kaposi's sarcoma after renal transplantation. Nephrol Dial Trransplant 1998; 13: 2768-73

9. Newstead CG. Assessment of risk of cancer after renal transplantation. Lancet 1993; 351:610-11

10. Hojo M, Morimoto M, Maluccio M. Cyclosporine induces cancer progression by a cell-autonomous mechanism. Nature 1999; 397:530-4

11. Vella JP, Mosher R, Sayegh MH. Kaposi's sarcoma after renal transplantation .N Engl J Med 1997; $336: 1761$

12. Hussein M M, Mooij J M , Roujouleh $\mathrm{H}$ MRegression of post-transplant Kaposi's sarcoma after replacing cyclosporine with mycophenolate mofetil.

Saudi J kidney Dis.Transpl 2001; 12 (1): 42-44.

13. Ganez E, Aguado S, Rodriguez M, etal. Kaposi's sarcoma after renal transplantation - disappearance aaafter reduction of immunosuppression \& reappearance 7 years later after start of mycophenolate mofetil treatment. Neprol Dial Transplant 1998;13:3279-80 .

14. Eberhard OK, Kliem V, Brunkhorst R. Five cases of Kaposi's sarcoma in kidney graft recipientspossible influence of the immunosuppressive therapy. Transplantation 1999; 67: 180-4.

15. Penn I. Sarcoma in allograft recipients. Transplantation 1995; 60:1485-91. 